Cahiers de recherches médiévales

Journal of medieval studies

$11 \mid 2004$

Figures mythiques médiévales auX $\mathrm{XIX} \mathrm{X}^{\mathrm{e}}$ et $\mathrm{XX} \mathrm{X}^{\mathrm{e}}$ siècles

\title{
L'écriture du Moyen-Âge dans Le Rêve de Zola
}

\section{Éléonore Reverzy}

\section{OpenEdition}

Journals

Édition électronique

URL : https://journals.openedition.org/crm/1803

DOI : $10.4000 / \mathrm{crm} .1803$

ISSN : 1955-2424

Éditeur

Honoré Champion

Édition imprimée

Date de publication : 15 décembre 2004

Pagination : 141-150

ISSN : 1272-9752

Référence électronique

Éléonore Reverzy, "L'écriture du Moyen-Âge dans Le Rêve de Zola », Cahiers de recherches médiévales

[En ligne], 11 | 2004, mis en ligne le 10 octobre 2007, consulté le 15 décembre 2022. URL : http:// journals.openedition.org/crm/1803 ; DOI : https://doi.org/10.4000/crm.1803

Ce document a été généré automatiquement le 15 décembre 2022.

Tous droits réservés 


\title{
L'écriture du Moyen-Âge dans Le Rêve de Zola
}

\author{
Éléonore Reverzy
}

« La vie telle qu'elle n'est pas, tous bons, honnêtes, heureux »

(Fol. 1 du Plan, Ms NAF 10323).

1 Le Rêve, en dépit de toutes les déclarations de Zola qui affirme à plusieurs reprises que le sujet est programmé dès les premiers plans, est en fait la réalisation d'un projet tardif. Il émane d'une volonté de surprendre le public et est donc, comme La Bête humaine immédiatement après, écrit en fonction d'une attente, qu'il s'agit en définitive de décevoir. La première phrase de l'Ébauche, qui est le stade liminaire du travail de conception chez Zola avant les Plans détaillés, les Fiches Personnages et les notes documentaires, est la suivante: "Je voudrais faire un livre qu'on n'attende pas de moi $^{1}{ }^{\prime}$. C'est dire que le premier souci du romancier est la réception de son roman, une réception qu'il cherche, en réfléchissant sur le statut générique de son œuvre, à modifier en tirant son lecteur de ses habitudes.

2 En 1887, le Manifeste des cinq a marqué en effet la fin d'un règne et la contestation d'un modèle, celui du roman naturaliste: pour Zola il s'agit de poursuivre son cycle et d'explorer parallèlement d'autres territoires génériques, celui du merveilleux dans Le Rêve («Il faudrait, pour première condition, qu'il pût être mis entre toutes les mains, même les mains des jeunes filles ») et celui du roman policier dans La Bête humaine. David Baguley a bien montré en quels termes se posait la question du générique pour les naturalistes dont la littérature est "foncièrement générique ${ }^{2}$ " malgré les propos des théoriciens du mouvement. Le Rêve programme manifestement les attendus du conte, comme l'atteste dans les Dossiers préparatoires, la référence fréquente au « prince charmant » espéré par Angélique.

3 C'est aussi que Le Rêve est fondamentalement un roman de la lecture et de la lectrice, je veux dire un roman où ce personnage occupe la position centrale et où la lecture qu'il pratique, celle de La Légende dorée particulièrement, est une mauvaise lecture, une lecture non pas critique mais « héroïque ${ }^{3}$ ». Dans Le Rêve, disparaît le personnage 
délégué du sens, celui chargé d'énoncer ou simplement d'incarner la leçon de l'œuvre, rôle traditionnellement dévolu $\mathrm{au}(\mathrm{x})$ médecin(s) ou à des figures féminines qui allégorisent la vie (Denise dans Au Bonheur des Dames, Pauline dans La Joie de vivre). C'est ce qui produit une déstabilisation du sens puisque le narrateur zolien recourt peu à la fonction auctoriale et évite d'adopter la position de surplomb qui lui permettrait d'orienter le point de vue du lecteur.

L'univers médiéval, omniprésent dans le roman, qui est l'un des plus anhistoriques des Rougon-Macquart, paraît d'abord surtout obéir à des contraintes d'ordre thématique et générique : il favorise la représentation de "l'inconnu, l'inconnaissable ${ }^{4}$ » et participe à la création d'un milieu irréel, caractéristique du conte. Le Moyen Âge est donc partout dans un roman qui est aussi, comme Le Ventre de Paris à d'autres titres ${ }^{5}$, une réponse au Notre-Dame de Paris de 1831 et une réflexion sur le symbole. C'est sous cet angle que je vais chercher à éclairer l'écriture du Moyen Âge dans le roman : la période médiévale est avant tout une mine où puiser une série de représentations, où chercher une esthétique (dans l'architecture comme dans la broderie), et une poétique du roman suivant un modèle allégorique, cher au romancier depuis le début de sa série ${ }^{6}$.

L'allégorie est en effet au cœur d'une poétique du roman qui suppose un double discours, ou qui suppose, pour être plus précise, un sens caché sous le sens littéral. Ainsi, en racontant la conquête de Plassans par les Rougon, dans La Fortune des Rougon, Zola prétend raconter le coup d'état de Louis-Napoléon III à Paris et les victimes des Rougon représentent toutes celles que la prise du pouvoir par Bonaparte a causées. C'est donc un travail de déplacement et de réduction, parfois apparenté à l'écriture burlesque, qu'opère le romancier dans la représentation de l'histoire ${ }^{7}$. Le déplacement est d'une autre nature lorsque le roman doit se charger de délivrer une signification, non pas historique, mais philosophique, ou lorsqu'il s'agit d'allégoriser la création ellemême (les Halles ou le grand magasin, images de l'œuvre). Dans Le Rêve, la cathédrale, les ruines du château des Hautecœur, l'atelier des Hubert sont autant de lieux qui d'une part assurent la médiévalisation ${ }^{8}$ du récit, d'autre part font signe vers l'œuvre, qu'elles en figurent des modèles ou des anti-modèles. De plus, le livre (La Légende dorée), le vitrail et la broderie constituent d'autres reflets de la création. Zola insiste beaucoup dans les Dossiers sur « l'artiste » que doit être Angélique9.

Dans un roman intitulé Le Rêve, il est clair, dès l'Ébauche, qu'il s'agit pour le romancier de représenter cette part $\mathrm{d}$ '« inconnaissable » et que là gît la difficulté :

«Le Rêve » serait le titre du volume, et c'est surtout ce qui me plaît. Je voudrais que le volume fût la partie du rêve dans la série, la fantaisie, l'envolée, l'au-delà. Et cela serait franc, puisque le titre avertirait le lecteur : «Voilà du rêve, je le dis, prenez-le comme tel ». Et alors, sans ironie trop, il faudrait y mettre la vie telle qu'elle n'est pas, telle qu'on la rêve : tous bons, tous honnêtes, tous heureux. Une vie idéale, telle qu'on la désire. Mais l'écueil de ça est de faire petit, plat et bête. On ne peut guère s'en sauver que par l'envolée, sans sortir toutefois de la simplicité. - Les éléments du problème à résoudre restent d'ailleurs toujours les mêmes : pureté, psychologie, au-delà ${ }^{10}$.

$7 \mathrm{Et}$, quelques folios plus loin: «Je finis quand les époux ressortent de l'église, la grand'porte de l'église large ouverte, et donnant sur le monde. Là, je m'arrête. La fin du rêve, l'entrée dans la réalité ${ }^{11}$ ».

8 La narration épouse donc le rêve dans sa durée et cesse quand il se confronte à la réalité, de même que le roman figure à l'intérieur du cycle, une pause, un temps d'arrêt, une parenthèse (il n'est plus question ni des Rougon-Macquart, ni des considérations 
sociales et des thèmes et motifs auxquels le romancier a accoutumé son lecteur, ni des lieux - Plassans, Paris - où se déroule la majeure partie des volumes). C'est ce qui lui confère une dimension particulièrement statique, très neuve pour le lecteur accoutumé aux drames que Zola dont la représentation du monde est fondamentalement darwinienne, se plaît à bâtir. Comme l'a relevé Colette Becker ${ }^{12}$, Le Rêve étonne par toute une série de limitations ( $\mathrm{du}$ nombre des personnages, des lieux, des intrigues secondaires) qui lui confère une forte concentration romanesque. Cette simplification, au moins apparente, participerait sans doute à l'esthétique du conte, d'autant que l'intrigue - une jeune fille pauvre qui attend le prince charmant et l'épouse après avoir franchi une série d'épreuves dont la mort - présente bien les caractéristiques de la légende et s'apparente en outre, vu les rôles que chacun joue sur l'échiquier (Saint Georges ou Jésus pour Félicien, Sainte Agnès pour Angélique) à un récit hagiographique ${ }^{13}$.

9 Mais c'est là ce qui pose un premier problème à Zola, celui déjà rencontré dans la conception de La Joie de vivre, l'écueil du hiératisme: en créant le personnage allégorique qui personnifie la joie de vivre, Pauline, Zola sait qu'il court de tels risques ${ }^{14}$ et il contourne la difficulté en axant sur Lazare l'action narrative - qui est celle de "l'émiettement", idée centrale du roman - et en donnant un défaut à Pauline, la jalousie, qu'elle combat et maîtrise peu à peu dans son fanatisme de charité. La personnification de la vertu qu'est à maints égards l'héroïne du Rêve connait dans une certaine mesure le même sort mais la dramatisation est d'une tout autre ampleur : il s'agit de montrer les passions héréditaires peu à peu corrigées et vaincues par l'influence du milieu, comme il s'agit d'inventer un conflit dans un milieu où tous sont "bons". C'est donc une nouvelle expérience de type naturaliste, suivant le patron fourni par la théorie même du Roman expérimental, que tente le romancier, et la métaphore végétale qu'il emploie, celle de la transplantation, est filée tout au long du récit et s'actualise en outre dans l'églantier, qu'Angélique a replanté et dont elle espère qu'il donnera des roses. L'églantier fonctionne comme ce que je nommerais un autoemblème, puisqu'il devient l'attribut et la projection symboliques de la jeune fille, à ses propres yeux ${ }^{15}$. À quoi sert pour autant le Moyen Âge, qui est à la fois si visible et si absent?

Bien sûr d'abord à dénaturaliser le récit, à accroître en conséquence la part de merveilleux dont il est lesté. De plus l'univers médiéval est une nouvelle couche de savoirs à explorer et à proposer au lecteur (l'ampleur des recherches menées par le romancier et conservées dans les Dossiers en témoigne) : les vitraux, le roman et le gothique, les légendes, tels sont, avec la broderie ou les grands rituels catholiques, les nouveaux champs parcourus dans une perspective didactique. Surtout, le Moyen Âge est secrété dans l'œuvre elle-même, par les personnages et devient, en ce qui regarde Angélique, un grand fantasme, le fondement même de son rêve.

11 Dans les notes qu'il prend sur la religion, Zola s'interroge particulièrement sur la grâce, à partir d'une citation de Renan :

Renan : «Il n'y a point de miracles dans ce monde ; le monde ne présente point de fait* particuliers, révélant des volontés particulières ». L'opinion contraire : nous vivons entourés de miracles, et les faits particuliers, contraires aux prétendues lois, nous débordent : phénomènes de magnétisme, d'hypnotisme, de vision à distance et de divination ([ill.] ?). Voilà mon au-delà. Mais pour moi ce ne sont que des forces inconnues de la matière - Ceux qui trouvent folle cette conception d'un monde mécanique régi par des ordres fixes. Ils croient aux miracles ${ }^{16}$. 
Cette question du miracle est intéressante car elle oppose le romancier à ses personnages, à Angélique surtout qui vit sur le mode du merveilleux, du fait de sa lecture de La Légende dorée, et qui s'identifie aux saintes. Celles qu'elle voit sur le porche de la cathédrale, dans les vitraux ou dans le livre de Voragine, pour relever de trois modes de représentation et de trois langages distincts, n'en génère pas moins la même adhésion et la même identification de la part de la jeune fille : Angélique en reste à la lettre, elle n'interprète pas et sa lecture n'est que de pur déchiffrement, ce qui, JeanLouis Cabanès l'a récemment montré ${ }^{17}$, renvoie au propos sur les dangers du symbole, omniprésent au XIX ${ }^{\mathrm{e}}$ siècle (Maury, Michelet, Flaubert). Surtout, les langages qu'elle tente de déchiffrer ressortissent tous de l'archaïque, du médiéval et en sont d'autant plus étranges que leur accès est difficile. Aussi Angélique, dans sa première lecture de la vieille édition de La Légende dorée, ne peut en déchiffrer les caractères gothiques et se contente des images qui figurent en regard du texte. C'est dire que c'est d'abord une imagerie $^{18}$ (illustrations du livre, statues du porche, saintes du vitrail ${ }^{19}$ ) qui la séduit et produit en elle cette entrée dans "l'inconnu» et cette foi au "miracl». Le mauvais lecteur est celui qui ne lève pas le voile des analogies et qui s'arrête aux images, sans percevoir qu'elles dissimulent autre chose : la lecture, telle que la pratique Angélique, pose en fait le problème de l'allégorie, ou plus exactement de l'allégorèse (son déchiffrement). Angélique ne s'intéresse ainsi jamais à la valeur morale que personnifient les saintes : la charité, l'humilité, la pénitence, ad libitum, même si elle imite inconsciemment leur martyre et pratique la vertu qu'elles illustrent avec le même excès (la charité notamment).

Le Moyen Âge fonctionne donc comme un détour qu'emprunte le romancier : il est l'archaïque, et en même temps il est un réservoir d'images, une sorte d'immense galerie où serait concentrée, voire concrétée la naïveté. Cette naïveté contamine le volume, qui gagne le romancier lui-même jusque dans les Dossiers préparatoires où il s'abandonne aux vertiges du romanesque et à tous les charmes de la romancie (les personnages principaux rassemblent sur eux la beauté, la grandeur d'âme, voire, pour les Hautecœur, la richesse ${ }^{20}$, les structures du romance ${ }^{21}$ sont reprises) et où il ne cesse de se mettre en garde contre l'ironie. L'ironie pourtant gît tout au fond du récit, au cœur même de son projet et, comme l'écrit $\mathrm{C}$. Becker, si «Le Rêve est une œuvre où le sens ne se fige jamais ${ }^{22}$ ", c'est bien à cette ironie tapie sous la mièvrerie et l'imagerie sulpicienne qu'elle doit cette instabilité signifiante. Dans ses Notes sur la religion, décidément précieuses, Zola oppose en effet le milieu et l'hérédité (c'est là, on s'en souvient, le conflit dont est chargé le personnage d'Angélique) et comprend que cette opposition peut s'énoncer par celle, théologique, de la grâce et du péché. Dès lors, il lui apparait indispensable de faire d'Angélique une " chrétienne primitive »:

Seulement, il faut remarquer que le milieu joue ici le rôle de la grâce, dans la théologie : il faut la grâce pour faire son salut, l'homme ne se sauve pas lui-même, si Dieu ne lui accorde pas la grâce ; et de même mes Rougon-Macquart ne résistent pas à leur hérédité, si le milieu ne vient la combattre. De même que mon Serge se désespérait lorsque la grâce l'abandonnait, il faudrait donc qu'Angélique entrât en angoisse dès qu'elle sentirait le milieu ne plus influer, n'être pas assez fort, disparaître. Ce milieu est aussi fait des légendes qu'elle a lues, de la foi qu'elle a bue dans cet air, de l'au-delà dans lequel elle baigne, de sa croyance aux miracles, du surnaturel en un mot. Tout cela est anti- rationaliste. Je me rencontre avec les chrétiens primitifs, Saint-Paul, Saint-Augustin, qui niaient le libre arbitre de l'homme plus ou moins. Mon hérédité [cl] c'est le péché originel d'Adam et d'Ève, transmis à leurs descendant*; et il [ne] n'y a plus que la grâce qui puisse le 
combattre, la grâce venue de Dieu, le surnaturel, la légende, la foi [au] à la vie des Saints et aux miracles. Donc dans mon bouquin, [ill.] tout l'au-delà, tout le surnaturel que je veux mettre dans le milieu, c'est à proprement parler la grâce, ce que Dieu envoie pour sauver mon héroïne; et si elle ne l'avait pas, elle ne se sauverait pas. Il faut donc faire d'Angélique une chrétienne primitive s'abandonnant aux mains de Dieu, disant que sans lui elle ne serait rien, ayant la foi [des], la croyance des premiers siècles. Tout est ramené à l'enfance dans ce qu'elle pense et dans ce qu'elle fait. Art archaïque, etc. Tout entière enfoncée dans sa Vie des Saints, comme vie morale. Et tirer une grâce de la Grâce. C'est le rêve aussi, elle est dans les extatiques, tout en l'air. Avec des luttes pourtant ${ }^{23}$. l'ignorance : le Moyen Âge est ce qui permet de dire cette ignorance, il correspond en somme à un stade primitif du développement mental et de l'intellection de l'univers. Il s'agit cependant d'une construction imaginaire du personnage lui-même, qui secrète en quelque sorte du médiéval, qui élabore un fantasme médiéval. Le romancier poursuit en effet :

Bien dire qu'en lisant la Vie des Saints, elle a vu qu'ils ne pouvaient rien pour le salut, si Dieu ne les soutenait pas, [leur] ne leur envoyait pas la grâce. Et c'est là que je la montre attendant tout [du] de la grâce, du milieu, du surnaturel de la légende qui l'entoure. - Cela me sert à poser toute mon interprétation de la grâce qui est le milieu- Ici, seulement, l'explication devient subtile, car dans mon idée le surnaturel du milieu est un effet réflexe de l'imagination, de la rêverie d'Angélique [lui] elle-même. Dans ma série, je ne puis admettre l'au-delà, l'inconnu que comme un effet de force* qui sont en [mon] nous dans la matière et que nous ne connaissons pas, simplement. Il faudrait donc montrer comment Angélique, avec ses désirs ignorés, son imagination nourrie de légendes, sa puberté s'épanouissant dans l'ignorance et dans le rêve, crée elle-même le milieu, l'au-delà, l'invisible, qui agit ensuite sur elle-même, par un effet de retour ${ }^{24}$.

C'est donc la génération d'un fantasme que raconte Zola dans Le Rêve, la manière dont un individu invente, pour son propre salut, un filtre qui est un véritable mode d'appréhension du monde, un moule dans lequel l'univers entier vient se fondre. Le Moyen Âge est ce moule: chargé de représentations, lesté d'images, il est la boîte à images, l'accélérateur de représentations dans un vaste va-et-vient qui va du Moyen Âge légendaire au Moyen Âge projeté sur l'univers ambiant. Zola conclut - et ce sera la conclusion du livre :

De sorte que le milieu, la prétendue grâce venue de [D] Dieu, viendrait de l'homme [po] pour améliorer l'homme. Cela rentrerait dans la théorie qu'il n'y a qu'illusion de nos sens, que nous créons le monde, que tout part de nous pour revenir à nous. Le rêve enfin. Et ce serait élargir le livre à la fin que de montrer ainsi que tout est un rêve, que chacun de nous n'est qu'une apparence qui disparaît après avoir cré/ er/é une illusion ${ }^{25}$.

En cela, sous ses dehors sulpiciens, Le Rêve est sans nul doute une terrible machine de guerre contre l'Église où s'énonce une critique de la croyance comme illusion : la foi n'est qu'une auto-suggestion et le catholicisme de fait placé entre Marx et Freud... L'ironie est donc bien cachée, puisqu'on peut lire - et on a de fait beaucoup lu ! - Le Rêve comme un "conte bleu ». La question de la réception que le romancier posait dès les premières pages de l'Ébauche est bien pendante.

17 Comme le relève Danielle Chaperon, Le Rêve ne parle pas seulement de consommation, mais aussi de production d'images ${ }^{26}$ : les Hubert sont brodeurs, Félicien peintre verrier et c'est de fait un va-et-vient qui s'opère entre les illustrations de La Légende et la broderie ou la restauration des vitraux. D'images à d'autres images, ce qui confirme la 
représentation du Moyen Âge comme réservoir d'images - ce que Zola avait pourtant dénoncé à propos de Notre-Dame de Paris et des romantiques en général qui n'avaient fait qu'employer un bric-à-brac médiéval et n'avaient montré de la période que quelques oripeaux, un attirail, un fatras ${ }^{27}$. C'est donc sur le terrain de l'esthétique qu'il convient de s'aventurer maintenant. La cathédrale pèse de son ombre sur tout le récit, comme sur le destin d'Angélique : figure à la fois maternelle et effrayante, elle tient du château des romans gothiques - et Zola a d'ailleurs un temps envisagé que ce soit le château des Hautecœur qui domine la vieille ville de Beaumont ${ }^{28}$ - et de la mère :

La cathédrale explique tout, a tout enfanté et conserve tout. Elle est la mère, la reine au milieu du petit tas des maisons basses, pareilles à une couvée abritée frileusement sous ses ailes de pierre. On n'y habite que par elle et pour elle; les industries ne travaillent, les boutiques ne vendent que pour la nourrir, la vêtir, l'entretenir, elle et son clergé ; et, si l'on rencontre quelques bourgeois, c'est qu'ils y sont les derniers fidèles des foules disparues. Elle bat au centre, chaque rue est une de ses veines, la ville n'a d'autre souffle que le sien $^{29}$.

Comment mieux dire qu'elle est à la fois cœur et vampire, qu'elle donne la vie et l'aspire? Les pages qui précèdent voient une petite fille inconnue se protéger de la neige sous son porche, se pétrifier peu à peu, et gagnée par le froid, saisie par la mort, devenir statue à son tour ${ }^{30}$. La cathédrale est mortifère et le livre qu'elle propose, qui ne raconte que des martyres de saintes, modèle par avance le destin de l'enfant : c'est sous ce même porche, qu'à l'excipit, Angélique mourra.

À ce livre de pierre répond le livret d'infamie que dissimule l'enfant et qu'elle finit par lâcher : c'est là l'autre récit de son destin, et c'est aussi son autre visage qui apparaît sous l'effet de la colère. Au visage de la "petite vierge de vitrail ${ }^{31}$ » avec ses "yeux couleur de violette, au long cou d'une grâce de lis » s'oppose " la face méchante, le cou sensuel [...] gonflé d'un flot de sang ${ }^{32} »$ de l'enfant trouvée. Ce serait, si nous suivons de nouveau le propos dissimulé dans les avant-textes, l'opposition de la grâce et du péché, le conflit du milieu et de l'hérédité. L'un et l'autre également mortifères en somme, sont représentés comme un ensemble de signes (les statues de la cathédrale, le langage des symboles vs la prose purement informative du livret, les données factuelles et biographiques) qui vont dominer la vie du personnage : la position de la maison des chasubliers, qui touche la cathédrale et le rangement du livret dans le tiroir du bahut de l'atelier - comme du registre et des titres de Pauline dans le secrétaire de La Joie de vivre - en sont par ailleurs des indices limpides.

La blancheur de la neige a une évidente fonction métatextuelle à l'ouverture du roman : page blanche que le romancier doit noircir, sans doute, d'autant que c'est sous le porche d'un monument que s'ouvre le récit, ce qui redouble encore l'effet de commencement. Mais précisément la cathédrale n'est que temporairement blanchie et s'offre en fait, ici, masquée, recouverte : le livre qu'elle constitue est déjà écrit, et la fin du chapitre qui décrit le même porche et les mêmes statues, après un faux dégel, montre désormais les saintes dans toute leur splendeur ${ }^{33}$. Le romancier se fixe ainsi un programme esthétique, celui de répondre au livre de pierre («Ceci tuera cela » est déjà récrit dans Le Ventre de Paris ${ }^{34}$ ) et de bâtir un autre ensemble symbolique, qui pourrait être cette œuvre feuilletée et profondément ironique, dans laquelle l'allégorie, prisée par le Moyen Âge, joue un rôle clef. L'insertion des citations de La Légende dorée dans le chapitre suivant où Angélique apprend à lire dans le vieux volume, confronte le lecteur à une langue hérissée et archaïque. 
Mais Angélique, peu à peu, fut curieuse de savoir au juste ce que représentaient les gravures. Les deux colonnes serrées du texte, dont l'impression était restée très noire sur le papier jauni, l'effrayaient par l'aspect barbare des caractères gothiques. Pourtant, elle s'accoutuma, déchiffra ces caractères, comprit les abréviations et les contractions, sut deviner les tournures et les mots vieillis; et elle finit par lire couramment, enchantée comme si elle pénétrait un mystère, triomphante à chaque nouvelle difficulté vaincue. Sous ces laborieuses ténèbres, tout un monde rayonnant se révélait. Elle entrait dans une splendeur céleste. [...] Seule, la Légende la passionnait, la tenait penchée, le front entre les mains, prise toute, au point de ne plus vivre de la vie quotidienne, sans conscience du temps, regardant monter, $d u$ fond de l'inconnu, le grand épanouissement du rêve ${ }^{35}$.

21 C'est ici tout un « travail de la citation ${ }^{36}$ » que mène le romancier, découpant et collant des morceaux d'histoires : d'un côté un modèle, celui de la cathédrale, construite au fil des siècles - et le Dossier conserve la trace de cette extension temporelle que le romancier juge nécessaire ${ }^{37}$-, de l'autre des fragments narratifs mis bout à bout et plus ou moins glosés par le narrateur :

La prospérité, la santé sont en mépris, la joie commence aux privations qui tuent le corps. Et c'est ainsi que, triomphants, ils vivent dans des jardins où les fleurs sont des astres, où les feuilles des arbres chantent. Ils exterminent des dragons, ils soulèvent des tempêtes et les apaisent, ils sont ravis en extase à deux coudées du sol. Des dames veuves pourvoient à leurs besoins pendant leur vie, reçoivent en rêve l'avis d'aller les ensevelir, quand ils sont morts. Des histoires extraordinaires leur arrivent, des aventures merveilleuses, aussi belles que des romans. Et, après des centaines d'années, lorsqu'on ouvre leurs tombeaux, il s'en échappe des odeurs suaves.

Puis, en face des saints, voici les diables, les diables innombrables. « Ilz vollerent souvent environ nous comme mousches et remplissent lair sans nombre. Lair est aussi plein de dyables et de mauvais esperitz: comme le ray du soleil est plein de athomes cest pouldre menue ». Et la bataille s'engage, éternelle ${ }^{38}$.

Le résumé, neutre, du texte de Voragine d'une part, la citation d'autre part contribuent à en accentuer l'étrangeté : pour Angélique ces récits ne sont pas l'objet d'une appréhension distante, mais sont abordés comme mimétiques. Les attributs qui accompagnent chacun des saints ${ }^{39}$ l'accoutument à vivre sur le mode symbolique comme s'il s'agissait du mode réel. Iconolâtre donc, Angélique participe en outre à la reproduction de ces images et brode «les mêmes symboles, les chiffres de Jésus et de Marie, le triangle entouré de rayons, l'agneau, le pélican, la colombe, un calice, un ostensoir, un cœur saignant sous les épines ${ }^{40}$ ». C'est ainsi qu'elle brode, pour la mitre de Monseigneur, une sainte Agnès dont l'image renvoie à la fois à la statue du porche, à « une vieille statue de bois » qui sert pour la procession du Miracle, et à la Légende de Voragine. Cette saturation des images ${ }^{41}$, à laquelle Angélique participe elle-même, fait du récit du Rêve un texte hérissé lui aussi, obscur et qui donne au lecteur cette impression qu'on lui cache quelque chose, que tout n'est pas si simple. Oui, Le Rêve est irritant, non à cause de la mièvrerie qu'il brasse, que parce que le secret qu'il dissimule ne se laisse pas saisir.

23 Cette poétique du secret, qui passe par le feuilletage du texte, par sa dimension allégorique (il parle de milieu et d'hérédité, problématique naturaliste, en feignant de parler de grâce et de péché) trouve sans doute son actualisation dans la figure même d'Agnès, qui est tout à la fois le double d'Angélique, « la gardienne de son corps ${ }^{42}$ » et cette vierge nue, voilée de sa seule chevelure. Ce jeu de la nudité et de la pudeur, que la jeune fille reproduit en brodant or sur or la chevelure d'Agnès, sous les yeux de 
Félicien, est au cœur d'une poétique qui montre et cache, qui exhibe son romanesque pour mieux camoufler son physiologique, qui claironne son merveilleux et tait son naturalisme: "un livre qu'on n'attende pas de moi». Car la chevelure de la sainte procède d'un « à rebours » de la mimesis ; « elle ne dissimule pas, elle laisse deviner ${ }^{43}$ " le désir d'Angélique, ce corps que la grâce, la foi aux miracles, la cathédrale, la broderie, la légende des « mortes heureuses », bref toute cette construction médiévale du roman, ont contraint, enfermé.

Zola avait envisagé, en préparant Le Rêve, l'histoire d'une jeune fille et d'un homme vieillissant, dans lequel il s'était projeté ${ }^{44}$, scénario qui sera celui du Docteur Pascal écrit après la rencontre avec Jeanne Rozerot: c'est en tout cas une histoire de désir qu'il prétendait alors raconter, cette histoire qu'il entreprend ensuite de couvrir de signes et de brouiller d'images, parmi lesquelles émerge cependant cette Agnès, comme une figure de l'œuvre.

\section{NOTES}

1.Fol. 217/1, Ms NAF 10323, Ébauche. Dans La Bête humaine, il voudra faire « un drame violent à donner le cauchemar à tout Paris ».

2.D. Baguley, Le Naturalisme et ses genres, Nathan, «Le texte à l'œuvre », 1995, p. 6. Voir également Zola et les genres, University of Glasgow French and German Publications, 1993.

3.Par lecture « hérö̈que », j'entends, à la suite de Chantal Pierre-Gnassounou, la lecture d'adhésion, qui ne prend aucune distance par rapport au récit. Voir Zola : les fortunes de la fiction, Nathan, «Le texte à l'œuvre », 1999, pp. 78-79.

4.Fol. 218/2, Ébauche (NAF 10323).

5.Je renvoie à ce propos à mon article : « Hugo dans Zola : le procès ", Hugo ou les frontières effacées, Yann Jumelais et Dominique Peyrache-Leborgne dir., Nantes, Pleins feux, 2002.

6.Ce travail se veut le prolongement de plusieurs articles, parmi lesquels « À l'exemple des Bonaparte : La Fortune des Rougon, genèse des Origines » (Zola à l'œuvre, Gisèle Séginger éd., PUS, 2003, p. 109-119) et « Allégorie et symbole dans Le Rêve de Zola » (Colloque « Symboles et symbolisme au XIX siècle » organisé à l'Université de Montpellier III par Pierre Citti et Alain Vaillant, à paraître).

7.J'ai étudié ce rapetissement de l'histoire et de la politique dans un article consacré à Son Excellence Eugène Rougon (" Histoire et politique dans Les Rougon-Macquart : Son Excellence Eugène Rougon ", Écritures XIXe siècle, volume coordonné par Christian Chelebourg, à paraître chez Minard).

8.J'utilise ce terme par référence au livre de Jean de Grandsaigne sur Proust : L'Espace combraysien. Monde de l'enfance et structure sociale dans l'œuvre de Proust, Minard, 1981 ( $2^{\mathrm{e}}$ partie).

9.Dans l'Ébauche il note : « Il faut qu'elle soit une artiste, que ses broderies soient des chefs-d'œuvre » (fol. 254/29).

10.Fol. 266/10-227/11 de l'Ébauche. 
11.Fol. 232/16 (ibid.).

12.C. Becker, « Le rêve d'Angélique », Les Cahiers naturalistes, $\mathrm{n}^{\circ} 76,2002$, pp. 7-23.

13.Je renvoie à ce propos à l'article de Jean-Louis Cabanès : «Imitation,

désymbolisation, pathologie de la croyance » (Littérature et pathologie, Max Milner éd., Presses universitaires de Vincennes, 1987, pp.133-148) et à sa thèse Le Corps et la maladie dans les récits réalistes (1856-1893), Klincksieck, 1991, p. 610 et sq.

14.« La grosse affaire est de mettre Pauline dans un drame. [...] Je ne puis changer le tempérament de Pauline, qui est une santé morale avant tout, un calme natif dans l'honneur et dans l'équilibre des vertus. Il faut donc que je la plante au milieu du drame comme j'ai planté Nana. Tout roulera autour d'elle, en agissant sur elle », note-t-il dans l'Ancien Plan de La Joie de vivre (NAF 10311, fol. 367/2, 368/3).

15. "Elle le taillait, l'arrosait ; il y repoussait plus droit, il y donnait des églantines plus larges, d'une odeur fine ; ce qu'elle guettait, avec sa passion habituelle, répugnant à le greffer pourtant, voulant voir si un miracle ne lui ferait pas porter des roses. Elle dansait à l'entour, elle répétait d'un air ravi : “C'est moi ! c'est moi !” " (Le Rêve, Gallimard, «La Pléiade », t. IV, pp. 840-841). Voir également p. 937 : «Vous aviez raison, mère, il n'est pas près de porter des roses ", au moment où son rêve s'écroule et où elle revient à l'humilité (cf. p. 932).

16.Fol. 186/1 des Notes sur la religion et la grâce (NAF 10324).

17.J.-L. Cabanès, " Rêver La Légende dorée », Les Cahiers naturalistes, n 76, 2002 pp. 45-47.

18.J'emploie ce terme par référence au livre de Philippe Hamon : Imageries. Littérature et image au XIXe siècle, Corti, 2001.

19.Dans un scénario qui figure dans l'Ébauche, Zola avait imaginé qu'Angélique soit adoptée par un garde-chasse et contemple « les images collées contre le mur, Pyrame et Thisbé » (fol. 225/9) : autre modélisation de son destin.

20.«Pendant que j'y suis, pourquoi ne pas me payer une fortune colossale? », s'interroge le romancier au sujet de Félicien (Ébauche, fol. 299/82).

21.Notamment la présence de deux personnages féminins (une blonde et une brune) et le schéma du récit en «Et puis » préféré en apparence à celui, réaliste, en « Donc » (voir sur ce point Northrop Frye dans L'Écriture profane. Essai sur les structures du romanesque, Circé, 1998 (1976)).

22.Art. cité, p. 23.

23.Fol. 190/5 à 192/7 des Notes sur la religion (NAF 10324).

24.Fol. 192/7 à 194/9.

25.Fol. 195/10.

26.Danielle Chaperon, «L'autre cathédrale, Le Rêve d'Émile Zola et de Carlos Schwabe ", La Cathédrale, Joëlle Prungnaud éd., Université Charles-de-Gaulle-Lille III, « Travaux et recherches ", 2001, pp. 99-115.

27.Là où un romancier réaliste aurait peint les foules en prières, toute la ferveur du Moyen-Âge, montré le personnel de la cathédrale, Hugo n'a décrit que des " gargouilles ", des décors pittoresques, une sorte de bric-à-brac médiéval, et appliqué les théories de la Préface de Cromwell dans le système de ses personnages, selon lequel on embellit « le sylphe par le gnome » et on entasse « les bouffonneries sur les sublimités » («Victor Hugo », Face aux romantiques, Henri Mitterand éd., Bruxelles, Complexe, 1989 , p. 96 et p. 108).

28.Pour éviter la ressemblance avec La Faute de l'abbé Mouret, « je voudrais donc remplacer l'église par un château et l'évêque par un seigneur, un noble très haut » (fol. 307/90 de l'Ébauche). 
29.Le Rêve, éd. citée, p. 826.

30.« Debout sur son pilier, [...] la statue de la vierge enfant avait la pureté blanche, le corps de neige immaculé, dans cette raideur immobile du froid, qui glaçait autour d'elle le mystique élancement de la virginité victorieuse. Et, à ses pieds, l'autre, l'enfant misérable, blanche de neige, elle aussi, raidie et blanche à croire qu'elle devenait de pierre, ne se distinguait plus des grandes vierges » (ibid., p. 817).

31.Ibid., p. 824.

32.Ibid., p. 821.

33. "C'était maintenant toute une glace, des robes transparentes, aux dentelles de verre, qui habillaient les vierges » (ibid., p. 825).

34. "Ceci tuera cela, le fer tuera la pierre » (Le Ventre de Paris, Les Rougon-Macquart, éd. citée, t. I, p. 799).

35.Le Rêve, p. 831.

36.Je fais référence à l'ouvrage d'Antoine Compagnon : La Seconde main ou le travail de la citation, Le Seuil, « Poétique », 1979.

37. "Histoire de l'église. Mais surtout le roman en bas et l'élancement du gothique en haut. Un mot des différentes époques de construction. » (fol. 70 des Plans).

38. Le Rêve, p. 832.

39." Saint Jean l'Aumônier donnant aux pauvres ; Saint Mathias brisant une idole ; Saint Nicolas, en évêque, ayant à sa droite des enfants dans un baquet; et toutes les saintes, Agnès, le col troué d'un glaive, Christine, les mamelles arrachées avec des tenailles, Geneviève, suivie de ses agneaux, Julienne flagellée, Anastasie brûlée, Marie l'Égyptienne faisant pénitence au désert, Madeleine portant le vase de parfum » (ibid., p. 830-831).

40.Ibid., p. 848 .

41.J.-L. Cabanès note : «Tout se passe, en effet, comme si, dans Le Rêve, roman qui mime une imagerie, le romancier, particulièrement iconophile, n'avait pu se passer d'images » ("Les chevelures de légende dans Le Rêve et Violaine la chevelue », Zola à l'œuvre, op. cit., pp. 147-158, p. 150).

42.Le Rêve, p. 840.

43.J.-L. Cabanès, art. précité, p. 152.

44. "J'avais donc trouvé ceci. Un homme de quarante ans, [qui se] n'ayant pas aimé, jusque-là dans la science, et qui se prend d'une passion pour une enfant de seize ans. » (Fol. 219/3 de l'Ébauche). Plus loin et entre parenthèses : « (Moi, le travail, la littérature qui a mangé ma vie, et le bouleversement, la crise, le besoin d'être aimé, tout cela à étudier psychologiquement) » (fol. 221/5-222/6).

\section{AUTEUR}

\section{ÉLÉONORE REVERZY}

Université Marc-Bloch Strasbourg-II 